Einladung zur Veranstaltung

\title{
Onkologische Bildgebung: Standards für Staging und Therapiekontrolle am 30. September 2017 in Berlin
}

Sehr geehrte Kolleginnen und Kollegen,

in der Onkologie nimmt die Bildgebung eine zentrale Position ein. Durch Einsatz verschiedener bildgebender Verfahren ist die Radiologie nicht nur in fast alle onkologischen Behandlungen direkt eingebunden, sie beeinflusst auch ganz entscheidend therapeutische Vorgehensweisen. Die Radiologie ermöglicht die genaue Beurteilung des Tumorstadiums, eines möglichen Therapieansprechens und ist auch in der Nachsorge wichtiger Partner der onkologisch tätigen Fächer. Auch die onkologische Bildgebung hat - wie die Therapie - in den letzten Jahren einen Wandel hin zur selektiveren, tumorspezifischen Bildgebung erfahren. Funktionelle bildgebende Verfahren ergänzen die klassischen morphologischen Daten und ermöglichen so eine genauere Charakterisierung von Tumoren sowie eine frühzeitigere Beurteilung des Therapieansprechens. Die AG Onkologische Bildgebung der Deutschen Röntgengesellschaft bietet Ihnen mit der Fortbildung „Onkologische Bildgebung: Standards für Staging und The-

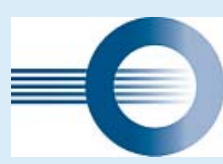

AC Onkologische Bildgebung

in der Deutschen Röntgengesellschaft

rapiekontrolle“ einen detaillierten Überblick über den aktuellen Standard der onkologischen Bildgebung am Beispiel des Mammakarzinoms, des Prostatakarzinoms sowie des Lymphoms. Gepaart mit Vorträgen zur modernen stadienadaptierten Therapie bieten wir Ihnen eine interdisziplinäre und praxisorientierte Fortbildung zur modernen onkologischen Bildgebung.

Wir freuen uns auf Ihre Teilnahme,

mit freundlichen Grüßen

Das Programm und die Anmeldung finden Sie auf onkologische-bildgebung.drg.de

ACHTUNG: Sie erhalten $50 \%$ Ermäßigung auf die Teilnahmegebühr der AG-Veranstaltung bei zusätzlicher Buchung des ICIS-Meetings! Bitte buchen Sie zuerst das ICIS-Meeting und danach die AG-Veranstaltung! Alle Infos zum ICIS-Meeting finden Sie hier: icimagingsociety.org.uk
Prof. Dr. H.-P. Schlemmer

PD Dr. Lale Umutlu

Prof. Dr. Gerald Antoch 